\title{
An Analysis of Factors Associated with Increased Perineal Descent in Women
}

\author{
Jina Chang, Soon Sup Chung \\ Department of Surgery, Ewha Womans University School of Medicine, Seoul, Korea
}

Purpose: Treatment of descending perineal syndrome is focused on personal etiology and on improving symptoms. However, the etiology of increased perineal descent $(\mathrm{PD})$ is unclear. Therefore, the aim of the present study was to evaluate factors associated with increased resting and dynamic PD in women.

Methods: From January 2004 to August 2010, defecographic findings in 201 female patients were reviewed retrospectively. Patient's age, surgical history, manometric results and defecographic findings were compared with resting and dynamic PD. Results: Age $(\mathrm{P}<0.01)$, number of vaginal deliveries $(\mathrm{P}<0.01)$ and resting anorectal angle $(\mathrm{P}<0.01)$ were correlated with increased resting $\mathrm{PD}$. Also, findings of rectoceles $(\mathrm{P}<0.05)$ and intussusceptions $(\mathrm{P}<0.05)$ were significantly correlated with increased resting $\mathrm{PD}$. On the other hand, increased dynamic $\mathrm{PD}$ was correlated with age $(\mathrm{P}<0.05)$, resting anal pressure $(\mathrm{P}<0.01)$ and sigmoidoceles $(\mathrm{P}<0.05)$. No significant correlation existed between non-relaxing puborectalis, history of pelvic surgery and increased PD. Also, no significant differences in PD according to the symptoms were observed.

Conclusion: Increased number of vaginal deliveries and increased resting rectoanal angle are associated with increased resting PD whereas increased resting anal pressure is correlated with increased dynamic PD. Older age correlates with both resting and dynamic PD. Defecographic findings, such as rectoceles and intussusceptions, are associated with resting PD, and sigmoidoceles correlated with dynamic PD. These results can serve as foundational research for understanding the pathophysiology and causes of increasing PD in women better and for finding a fundamental method of treatment.

Keywords: Perineum; Women; Defecography; Manometry

\section{INTRODUCTION}

Descending perineum syndrome was observed in patients with chronic constipation and was first defined as a relaxation of the pelvic floor by Parks et al. [1] in 1966. While the cause of increased perineal descent is believed to be excessive straining upon defecation, excessive straining causes the anterior rectal wall to protrude towards the anal canal, in turn inducing incomplete defecation and a weakness of the pelvic floor muscle. In addition, such in-

Received: July 12,2012 - Accepted: August 2, 2012

Correspondence to: Soon Sup Chung, M.D.

Department of Surgery, Ewha Womans University Mokdong Hospital,

1071 Anyangcheon-ro, Yangcheon-gu, Seoul 158-710, Korea

Tel: +82-2-2650-2517, Fax: +82-2-2644-7984

E-mail: colonclinic@ewha.ac.kr

(C) 2012 The Korean Society of Coloproctology

This is an open-access article distributed under the terms of the Creative Commons Attribution NonCommercial License (http://creativecommons.org/licenses/by-nc/3.0) which permits unrestricted noncommercial use, distribution, and reproduction in any medium, provided the original work is properly cited. complete defecation results in more excessive straining, and the weakened pelvic floor results in increased perineal descent, resulting in a vicious cycle $[1,2]$. Other authors have noted that the abnormal descent of the perineum not only causes constipation, but also frequently causes fecal incontinences, anal pain, and other symptoms [3-5].

For the management of descending perineum syndrome, surgical treatment has shown little efficacy, leading to the administration of various forms of conservative treatments, such as changes in diet, regulating defecation habits, use of purgatives, and biofeedback treatment. According to the study of Hur et al. [6], biofeedback has an approximately $92 \%$ success rate, shows symptomatic improvement and can be considered to be the most suitable method of treatment. In addition, D'Amico and Angriman [7] argued that a complete recovery from descending perineum syndrome was difficult to expect and that treatment should focus on improving symptoms according to the individual patient. From such a perspective, an analysis of the causes of the descending perineum syndrome is important. Causes in women are known 
to be associated with the number of vaginal deliveries, cases of obstructed labor, and anorectal surgery $[8,9]$ while rectoceles and intussusceptions are also known to induce excessive straining, causing constipation and resulting in a descent of the perineum. In addition, Pucciani et al. [10] showed that a total abdominal hysterectomy was also associated with perineal descent in women. Within such a context, we aimed to investigate the association between various factors and increased perineal descent in women.

\section{METHODS}

\section{Study subjects}

The subjects of this study were women with defecation disorder visiting the Department of Surgery, Ewha Womans University Mokdong Hospital, from January 1, 2004 to August 30, 2010. Patients showed symptoms of constipation, fecal incontinence, and anal pain. Tests were performed with the use of defecography.

\section{Radiologic examinations}

Defecography

For subject patients, defecography was administered, and the width of the anorectal angle and the perineal descending movement were measured. Without preparation, patients were placed in a left lateral position, and a Foley catheter was inserted through the anus. Fifty $\mathrm{mL}$ of barium sulfate suspension was injected into the rectum by using an enema syringe connected to a catheter, and the contrast medium paste was injected until the subject had an urge to evacuate. The contrast medium paste was obtained by mixing potato starch and barium with water. The contrast medium paste had the consistency of normal stool or more fluid to permit ease of injection into rectum. The patient was placed on a fluoroscopic toilet laterally and asked to gently defecate. The examination was performed by filming the dynamics of defecation step by step with short radioscopic sequences and radiographs. The phases of defecography are 1) during rest with filled anal bulb, 2) during maximum contraction of the anal sphincter and the pelvic floor muscles, 3) during straining without evacuation, 4) during evacuation, and 5) during rest when evacuation is completed. The patient must be instructed to empty the rectum completely and without interruption.

For the anorectal angle, the posterior anorectal angle, which is the angle between the anal canal axis and a line parallel to the posterior rectal wall, was used. For the width of the perineal descending movement, the vertical distance from the pubococcygeal line, which was from the top of the symphysis pubis to the bottom of the coccyx, to the point of the anorectal connection was measured (Fig. 1), while the dynamic perineal descent was defined by subtracting the resting phase value from the defecation value. In addition, rectoceles, intussusceptions or sigmoidoceles were diagnosed through defecography. Non-relaxing puborectalis was also assessed. Non-relaxing puborectalis indicates that upon attempting defecation, puborectalis relaxation does not occur, causing an indentation of the puborectalis. It, also, prevents the anorectal angle from widening or causing an increase, and cases where the anal canal did not open were diagnosed as cases of non-relaxing puborectalis.

\section{Anorectal manometry}

Some patients underwent anorectal manometry. The anorectal manometer consisted of an 8-channel water perfusion pump system (PIP-4-8 SS, Mui Scientific Inc., Ontario, Canada) and a balloon-attached catheter (Zinetics Manometric Catheter, Medtronic Inc., Minneapolis, MN, USA). The pressure measured through the catheter was converted into minute electric currents and recorded on a computed polygraph (Insight Manometry System S98-2000P and BioVIEW Analysis, Sandhill Scientific Inc., Highlands Ranch, CO, USA). The Patient was placed in the lateral position without preparation, and the catheter was inserted through the anus. The channel of catheter was positioned $6 \mathrm{~cm}$ distally from the anus, and the resting, squeezing and pushing phase pressures were measured. As the catheter was being removed by $1 \mathrm{~cm}$ sequentially, the pressure was measured using the same method. The mean resting pressure and the maximal voluntary contraction pressure were calculated. The mean squeezing pressure was defined by subtracting the mean resting pressure from the maximal voluntary contraction pressure.

\section{Data collection and statistical analysis}

The ages of the patients, number of vaginal deliveries, surgical histories and radiologic results were reviewed through medical records and analyzed retrospectively. Statistical significance was verified through the student t-test, Pearson correlation test and analysis of variance test while all analyses were conducted through SPSS ver. 16.0 (SPSS Inc., Chicago, IL, USA). Results with a P-value

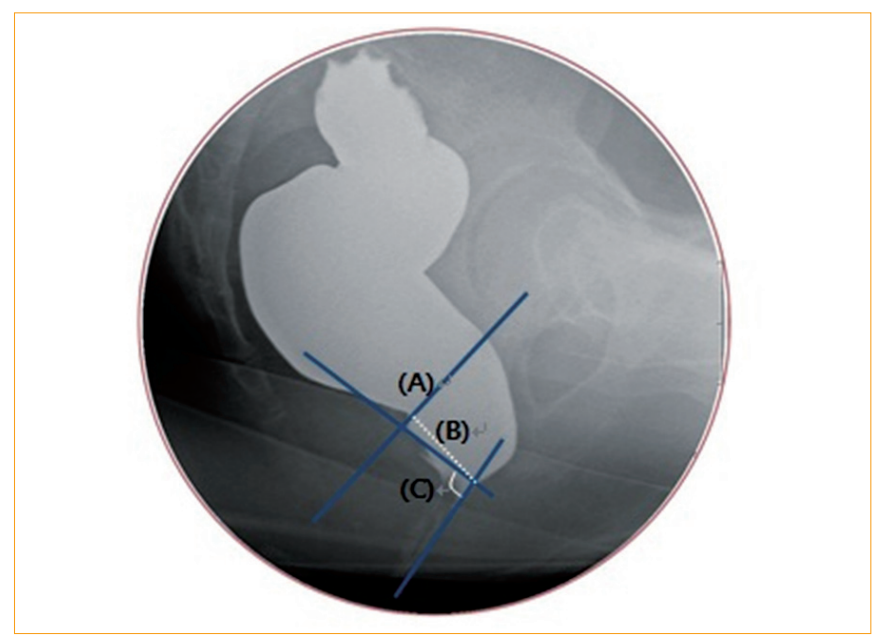

Fig. 1. Technique used for the measurement of perineal descent and anorectal angle; (A) pubococcygeal line, (B) perineal descent, (C) anorectal angle. 
under 0.05 were seen to have statistical significance.

\section{RESULTS}

\section{Patients' characteristics}

There were 201 patients with a mean age of $50.4 \pm 15.4$ years (range, 18 to 81 years). As for the major symptoms for the patients, 127 patients (63.2\%) showed constipation, 49 patients (24.4\%) showed fecal incontinence, 11 patients $(5.5 \%)$ had anal pain, and 14 patients $(6.9 \%)$ exhibited other symptoms. The mean number of vaginal deliveries was $1.84 \pm 1.44$ (range, 0 to 5 ) (Table 1 ).

\section{Defecographic findings}

As to the mean anorectal angle measured in defecography, in the resting phase, it was $103.7 \pm 15.2$ degrees, in the squeezing phase, it was $86.9 \pm 18.1$ degrees, and in the defecation phase, it was 122.5

Table 1. Patients' characteristics

\begin{tabular}{lc}
\hline Characteristic & Value \\
\hline Age (yr) & $50.4 \pm 15.4(18-81)$ \\
No. of vaginal deliveries (time) & $1.84 \pm 1.44(0-5)$ \\
Symptom & \\
Constipation & $127(63.2)$ \\
Fecal incontinence & $49(24.4)$ \\
Anal pain & $11(5.5)$ \\
Other & $14(6.9)$ \\
Finding on defecography & \\
Non-relaxing puborectalis & $124(61.7)$ \\
Rectocele & $138(68.3)$ \\
Intussusception & $41(20.4)$ \\
Sigmoidocele & $49(24.4)$ \\
\hline
\end{tabular}

Values are presented as Mean \pm SD (range) or number (\%).

Table 2. Anorectal angles and perineal descents on defecographic findings

\begin{tabular}{lc}
\hline Defecographic finding & Mean \pm SD (range) \\
\hline Anorectal angle $\left(^{\circ}\right)$ & $103.7 \pm 15.2(50.0-140.0)$ \\
\hline Resting & $86.9 \pm 8.1(35.0-135.0)$ \\
Squeezing & $122.5 \pm 18.3(75.0-163.0)$ \\
\hline Pushing & $18.8 \pm 16.3(-20.0-69.0)$ \\
\hline Dynamic change & \\
Perineal descent $(\mathrm{cm})$ & $5.5 \pm 1.3(2.8-11.4)$ \\
Resting & $4.7 \pm 3.2(1.5-9.6)$ \\
Squeezing & $7.0 \pm 1.8(2.9-13.5)$ \\
Pushing & $1.5 \pm 1.3(-2.0-5.5)$ \\
\hline Dynamic change & \\
\hline
\end{tabular}

\pm 18.3 degrees. The mean dynamic anorectal angle, the difference between the angles for the defecation and the resting phase, was $18.8 \pm 16.3$ degrees. The mean width of the perineal descent was $5.5 \pm 1.3 \mathrm{~cm}$ for the resting phase, $4.7 \pm 3.2 \mathrm{~cm}$ for the squeezing phase, and $7.0 \pm 1.8 \mathrm{~cm}$ for the defecation phase; the dynamic perineal descent was $1.5 \pm 1.3 \mathrm{~cm}$ (Table 2). In addition, there were 124 cases $(61.7 \%)$ of non-relaxing puborectalis syndrome, 138 cases $(68.3 \%)$ of rectoceles, 41 cases $(20.4 \%)$ of intussusceptions and 49 cases $(24.4 \%)$ of sigmoidoceles.

\section{Correlation between perineal descent and factors}

We analyzed factors that correlated with the resting phase of perineal descent in patients. Age, number of vaginal deliveries, and the size of the resting-phase anorectal angle showed significant correlations while the resting-phase or the squeezing-phase anal pressure did not have a statistically significant correlation (Table 3). Within the correlation analysis between the dynamic perineal descent and factors, age and anal pressure of the resting phase had a significant correlation (Table 4).

In addition, the perineal descent was compared between patient groups that showed non-relaxing puborectalis, rectoceles, intussusceptions and sigmoidoceles and a group of normal patients. Also, the group of patients with a history of having once received perineal surgery was compared with the group of patients with no history of perineal surgery. The groups that showed a statistically significant difference within resting-phase perineal descent were the groups with rectoceles and intussusceptions. The resting-phase perineal descents for the groups with rectoceles and intussusceptions were significantly larger than it was for the normal group, and those differences were statistically significant. A comparison of the dynamic perineal descents showed a statistically significant

Table 3. Correlations between perineal descent at rest and factors

\begin{tabular}{lcc}
\hline Factor & Correlation coefficient & P-value \\
\hline Age & 0.216 & $<0.01$ \\
No. of vaginal deliveries & 0.545 & $<0.01$ \\
Anorectal angle at rest & 0.201 & $<0.01$ \\
Anal pressure at rest & -0.049 & NS \\
Anal pressure at squeezing & -0.007 & NS \\
\hline
\end{tabular}

NS, non-specific.

Table 4. Correlations between dynamic perineal descent and factors

\begin{tabular}{lcc}
\hline Factor & Correlation coefficient & P-value \\
\hline Age & -0.144 & $<0.05$ \\
No. of vaginal deliveries & -0.099 & NS \\
Anorectal angle change & 0.129 & NS \\
Anal pressure at rest & 0.299 & $<0.01$ \\
Anal pressure at squeezing & 0.070 & NS \\
\hline
\end{tabular}

NS, non-specific. 
larger result in the patient group with sigmoidoceles (Table 5).

\section{Difference in perineal descent with symptoms}

There was no significant difference in either the resting or the dynamic perineal descent between the three groups, although the mean resting perineal descent of the fecal incontinence group was larger than it was for the other groups (Table 6).

\section{DISCUSSION}

Descending perineum syndrome, one of the functional causes of idiopathic chronic constipation, can be understood to be a relaxation of the pelvic floor resulting from excessive straining upon continuous defecations. Consequently, while this is a cause of constipation, it reversely is also a phenomenon furthered due to chronic constipation. Excessive perineal descent also results in anorectal pain or fecal incontinence.

Table 5. Correlations between perineal descent and other defecographic findings, as well as surgical history

\begin{tabular}{|c|c|c|c|c|}
\hline \multirow[t]{2}{*}{ Factor } & \multicolumn{2}{|c|}{$\begin{array}{l}\text { Resting perineal } \\
\text { descent }(\mathrm{cm})\end{array}$} & \multicolumn{2}{|c|}{$\begin{array}{l}\text { Dynamic perineal } \\
\text { descent }(\mathrm{cm})\end{array}$} \\
\hline & Mean \pm SD & P-value & Mean \pm SD & P-value \\
\hline NRPR & & NS & & NS \\
\hline Absent & $5.54 \pm 1.33$ & & $1.76 \pm 1.40$ & \\
\hline Present & $5.53 \pm 1.35$ & & $1.36 \pm 1.23$ & \\
\hline Rectocele & & $<0.05$ & & NS \\
\hline Absent & $5.12 \pm 1.02$ & & $1.51 \pm 1.45$ & \\
\hline Present & $5.72 \pm 1.42$ & & $1.52 \pm 1.24$ & \\
\hline Intussusception & & $<0.05$ & & NS \\
\hline Absent & $5.42 \pm 1.22$ & & $1.48 \pm 1.32$ & \\
\hline Present & $5.98 \pm 1.67$ & & $1.65 \pm 1.28$ & \\
\hline Sigmoidocele & & NS & & $<0.05$ \\
\hline Absent & $5.65 \pm 1.48$ & & $1.67 \pm 2.55$ & \\
\hline Present & $5.75 \pm 1.55$ & & $1.72 \pm 1.08$ & \\
\hline History of pelvic surgery & & NS & & NS \\
\hline Absent & $5.06 \pm 0.90$ & & $1.72 \pm 0.76$ & \\
\hline Present & $5.38 \pm 0.79$ & & $1.48 \pm 0.88$ & \\
\hline
\end{tabular}

NRPR, non-relaxing puborectalis; NS, non-specific.
The degree of perineal descent can be measured by administering defecography and defines the perpendicular distance from the anorectal connection, in other words, from the contact point where the distal rectal posterior line and the central axis of the anus canal meet to the pubococcygeal line $[11,12]$. However, as the measurement of the anorectal angle or perineal descent within defecography has poor reproducibility and differences exist between examiners, objective measurement through identical standards is important [13]. In addition, the definition of a significant increase of the perineal descent differs by author. In the case of Landmann and Wexner [14], an abnormal increased perineal descent was diagnosed in instances where the resting phase was over $4 \mathrm{~cm}$ and the dynamic perineal descent was over $3 \mathrm{~cm}$, and Parks et al. [1] diagnosed a significant increase when the resting phase was over $3 \mathrm{~cm}$ and dynamic perineal descent was also over $3 \mathrm{~cm}$ while Kim et al. [15] diagnosed cases of significant increase when the resting phase was over $5 \mathrm{~cm}$ and the dynamic perineal descent was over $4 \mathrm{~cm}$.

The perineal descent observed in a defecography has generally been seen as a phenomenon occurring due to various diseases that cause pelvic outlet obstruction rather than a disease occurring independently $[3,16]$, and clear explanations of descending perineum syndrome or related factors have yet to be given. Many previous studies attribute the cause to a weakening of the pelvic floor caused by external damage to the perineum or by trauma to the pudendal nerve caused by delivery in women $[9,14,17,18]$. In addition, there have also been opinions that it is related to surgical history, such a hysterectomy and repair of rectoceles and cystoceles [18]. In the present study, the association between the past history of patients and the amount of perineal descent was investigated. Increasing age, increasing number of vaginal deliveries, and increasing resting-phase perineal descent showed a statistically significant association, but surgical history did not show any correlation. Within research on association with dynamic perineal descent, while a relationship was shown with increasing age, no relationship was shown with number of vaginal deliveries or surgical history. In addition, Ahn et al. [19] reported that defecography for patients with defecation disorders showed various overlapping findings, particularly numerous cases of excessive perineal descent being accompanied by rectoceles, non-relaxing puborectalis or sigmoidoceles. In that study, however, no such significant relationships were discovered. The results of the present study showed a statistically-significant correlation between rest-

Table 6. Differences in perineal descent according to the symptoms of the patients

\begin{tabular}{|c|c|c|c|c|c|}
\hline \multirow{2}{*}{ Symptom } & \multirow{2}{*}{ No. } & \multicolumn{2}{|c|}{ Resting perineal descent (cm) } & \multicolumn{2}{|c|}{ Dynamic perineal descent (cm) } \\
\hline & & Mean \pm SD & P-value & Mean \pm SD & P-value \\
\hline Fecal incontinence & 49 & $5.84 \pm 1.44$ & 0.073 & $1.30 \pm 1.19$ & 0.411 \\
\hline Constipation & 127 & $5.49 \pm 1.33$ & & $1.58 \pm 1.34$ & \\
\hline Other & 25 & $5.12 \pm 1.06$ & & $1.61 \pm 1.35$ & \\
\hline Total & 201 & $5.54 \pm 1.34$ & & $1.51 \pm 1.31$ & \\
\hline
\end{tabular}


ing-phase perineal descent and resting anorectal angle while a statistically significant increase in resting-phase perineal descent was observed in patients with rectoceles or intussusceptions. On the other hand, the increase in dynamic perineal descent had no statistically significant relationship with diagnosis found through defecography except sigmoidoceles.

In addition, regarding the hypothesis that there was a relationship between the neuropathy of the puborectalis and descending perineum syndrome, the increased perineal descent might have occurred due to a weakening of the pelvic floor caused by perineal nerve damage. Research findings have indicated that patients showing signs of descending perineum syndrome exhibit nerve conduction delays on pudendal nerve terminal motor latency (PNTML) tests and that these findings have a significant relationship [20], but other authors have concluded the opposite by arguing that there is no significant relationship [21]. In addition, Lee and Park [17] suggested that increased perineal descent could be due to pudendal nerve damage following vaginal delivery, but abnormalities as shown on PNTML test was recovered within 2 months and increased perineal descent could continue for 6 months. The findings of the two tests could be different depending on the timeframe of clinical observation.

The PNTML test was not included in the present study. However, the correlation between anal pressure and nerve or sphincter damage was investigated. The resting-phase perineal descent had no relationship with either the resting or the squeezing anal pressure. However, the dynamic perineal descent had a significant relationship with the resting anal pressure. This result suggests that nerve injury could have an effect on dynamic perineal descent, but the reason for the different resting perineal descents is not clear.

The cause of increased perineal descent, excessive straining during defecation, weakened perineal muscle and excess perineal descent, are believed to make a vicious cycle. During the cycle, the perineal muscle would be weakened more, and fecal incontinence would be present as a late symptom of the cycle. Therefore, we suppose that patients with symptoms of fecal incontinence may have larger perineal descent. In the present study, the mean resting-phase perineal descent of patients with fecal incontinence was larger than it was for patients with other symptoms, but the differences were not statistically significant.

As mentioned before, rather than occurring independently, most cases of increased perineal descent are accompanied by various diseases that cause the onset of defecation disorder. As such, most forms of treatment focus on easing the symptoms of defecation disorder or remedying diseases believed to be a direct cause of the increased perineal descent rather than on the increased perineal descent itself. Cundiff et al. [22] reported that within descending perineum syndrome patients with accompanying vaginal prolapse, the results of clinical observations of 11 patients who underwent abdominal sacral colpoperineopexy showed improved symptoms in 8 patients, with perineal descent returning to normal ranges. Most studies, however, have argued that descending perineum syn- drome must be improved through conservative treatment rather than surgical fixation and have reported biofeedback to be particularly effective. Such efficacy of biofeedback resulted in improved symptoms regardless of constipation or fecal incontinence [23-25]. However, because the effects of such treatments are clear in the early stages where the increase in perineal descent is not severe, early treatment of the disorder through adequate examinations of patients with defecation disorder is important [18]. However, biofeedback focuses on improving symptoms rather than being a fundamental treatment. Consequently, studies on more fundamental forms of treatment are required for patients who have continuing or recurring symptoms after treatment.

The focus of the present study was to reveal factors associated with the increasing perineal descent frequently found in female patients with defecation disorders. The results of the study showed a statistically significant difference in factors associated with increased resting and dynamic perineal descent. The results show that with a better understanding of the pathophysiology of descending perineum syndrome, a fundamental method of treatment can be achieved. Additional studies are necessary to address factors not included in the present study so that a better understanding of relevant medical practices can be achieved.

\section{CONFLICT OF INTEREST}

No potential conflict of interest relevant to this article was reported.

\section{REFERENCES}

1. Parks AG, Porter NH, Hardcastle J. The syndrome of the descending perineum. Proc R Soc Med 1966;59:477-82.

2. Hardcastle JD. The descending perineum syndrome. Practitioner 1969;203:612-9.

3. Henry MM, Parks AG, Swash M. The pelvic floor musculature in the descending perineum syndrome. Br J Surg 1982;69:470-2.

4. Bartolo DC, Read NW, Jarratt JA, Read MG, Donnelly TC, Johnson AG. Differences in anal sphincter function and clinical presentation in patients with pelvic floor descent. Gastroenterology 1983;85:68-75.

5. Kim SH, Hwang YH, Choi KP. A role of anorectal physiologic study for the diagnosis of chronic constipation. J Korean Soc Coloproctol 2000;16:231-8.

6. Hur LN, Hwang YH, Jung YH. Outcome and predictors of success of biofeedback for descending perineum syndrome. J Korean Soc Coloproctol 2007;23:145-51.

7. D’Amico DF, Angriman I. Descending perineum syndrome: iatrogenic or spontaneous pathology? Chir Ital 2000;52:625-30.

8. Snooks SJ, Setchell M, Swash M, Henry MM. Injury to innervation of pelvic floor sphincter musculature in childbirth. Lancet 1984;2:546-50.

9. Andromanakos N, Skandalakis P, Troupis T, Filippou D. Constipation of anorectal outlet obstruction: pathophysiology, evalua- 
tion and management. J Gastroenterol Hepatol 2006;21:638-46.

10. Pucciani F, Boni D, Perna F, Bassotti G, Bellini M. Descending perineum syndrome: are abdominal hysterectomy and bowel habits linked? Dis Colon Rectum 2005;48:2094-9.

11. Ikenberry S, Lappas JC, Hana MP, Rex DK. Defecography in healthy subjects: comparison of three contrast media. Radiology 1996;201:233-8.

12. Mahieu P, Pringot J, Bodart P. Defecography: II. Contribution to the diagnosis of defecation disorders. Gastrointest Radiol 1984;9: 253-61.

13. Ferrante SL, Perry RE, Schreiman JS, Cheng SC, Frick MP. The reproducibility of measuring the anorectal angle in defecography. Dis Colon Rectum 1991;34:51-5.

14. Landmann RG, Wexner SD. Paradoxical puborectalis contraction and increased perineal descent. Clin Colon Rectal Surg 2008;21: $138-45$.

15. Kim KR, Kim YS, Chung SS, Ahn EJ, Oh SY, Park UC, et al. Investigation of defecographic findings in patients with pelvic outlet obstructive disease. J Korean Soc Coloproctol 2005;21:376-83.

16. Park UC, Choi SK, Piccirillo MF, Verzaro R, Wexner SD. Patterns of anismus and the relation to biofeedback therapy. Dis Colon Rectum 1996;39:768-73.

17. Lee SJ, Park JW. Follow-up evaluation of the effect of vaginal delivery on the pelvic floor. Dis Colon Rectum 2000;43:1550-5.

18. Harewood GC, Coulie B, Camilleri M, Rath-Harvey D, Pemberton JH. Descending perineum syndrome: audit of clinical and laboratory features and outcome of pelvic floor retraining. Am J Gastroenterol 1999;94:126-30.

19. Ahn EJ, Jeong GY, Cheon SH, Lee EJ, Oh SY, Chung SS, et al. The clinical and physiologic characteristics of patients with pelvic outlet obstructive disease. J Korean Soc Coloproctol 2005;21:362-9.

20. Bartolo DC, Roe AM, Mortensen NJ. The relationship between perineal descent and denervation of the puborectalis in continent patients. Int J Colorectal Dis 1986;1:91-5.

21. Jorge JM, Wexner SD, Ehrenpreis ED, Nogueras JJ, Jagelman DG. Does perineal descent correlate with pudendal neuropathy? Dis Colon Rectum 1993;36:475-83.

22. Cundiff GW, Harris RL, Coates K, Low VH, Bump RC, Addison WA. Abdominal sacral colpoperineopexy: a new approach for correction of posterior compartment defects and perineal descent associated with vaginal vault prolapse. Am J Obstet Gynecol 1997; 177:1345-53.

23. Enck P. Biofeedback training in disordered defecation. A critical review. Dig Dis Sci 1993;38:1953-60.

24. Guillemot F, Bouche B, Gower-Rousseau C, Chartier M, Wolschies E, Lamblin MD, et al. Biofeedback for the treatment of fecal incontinence. Long-term clinical results. Dis Colon Rectum 1995; 38:393-7.

25. Ko CY, Tong J, Lehman RE, Shelton AA, Schrock TR, Welton ML. Biofeedback is effective therapy for fecal incontinence and constipation. Arch Surg 1997;132:829-33. 